significant. GPC3 and E-cadherin positivity rates were significantly interrelated in NMA, but not MA groups. In NMA group, there was no significant relation between GPC3, E cadherin expressions and the clinicopathological features. Conversely, high E-cadherin expression in MA cases was associated with old age, fungating tumor configuration, mucoid adenocarcinoma rather than signet ring carcinoma subtypes and negative intratumoral lymphocytic response. Neither GPC3 nor E-cadherin expression showed a significant impact on disease-free survival or overall survival.

Conclusion: GPC3 and E-cadherin expressions are not independent prognostic factors in CRC. However, expressions of both are significantly interrelated in NMA patients, suggesting an excellent interplay between both, in contrast to MA. Further molecular studies are needed to explore the relationship between GPC3 and E-cadherin in colorectal carcinogenesis.

\section{DIFFUSE COLLAGENOSIS OF THE GASTROINTESTINAL TRACT - A CLINICOPATHOLOGICAL STUDY OF 10 CASES}

Carrie Gallivan $^{1}$, Ian Brown ${ }^{1,3}$, Namrata Setia ${ }^{2}$, Christophe Rosty $^{3}$, Gregory Y. Lauwers ${ }^{2}$

${ }^{1}$ Pathology Queensland, Brisbane, Australia; ${ }^{2}$ Gastrointestinal Pathology Service, Massachusetts General Hospital and Harvard Medical School, Boston, MA, USA; and ${ }^{3}$ Envoi Pathology, Brisbane, Australia

Background: Pathological subepithelial collagen deposition (collagenosis) is described in all parts of the glandular gastrointestinal tract. In many cases an aetiology is not established. Immune dysfunction and medications are responsible for some cases. Chronic diarrhoea is the most frequent presentation in adults. Generally only one site is involved. Collagenosis affecting both upper (stomach and/or duodenum) and lower (colon \pm ileum) gastrointestinal tract (diffuse collagenosis, DC) is rare and has not been systematically studied.

Design: Cases of DC from the authors' institution were reviewed. Recorded clinical data included presenting symptoms, medication use and presence of co-morbidities. Histological parameters included maximum collagen band thickness, intraepithelial lymphocyte (IEL) density, degree of lamina propria chronic inflammation, extent of eosinophil and neutrophil infiltration and degree of villous architectural change (if applicable). Results: There were 10 cases ( 9 females, 1 male) with age range of 30-74 (mean 62). Diarrhoea was the presenting symptom in eight cases. Gluten-sensitive enteropathy was present in two patients and other autoimmune disease in three patients. In available data, there was no consistent pattern of medication use prior to development of symptoms. Follow-up ranged from 1 month to 3 years, with one case showing collagen disappearance. In available data, treatment included prolonged Budesonide therapy. Collagen deposition spared the duodenum in three patients.

Conclusions: DC is a rare condition most commonly presenting with diarrhoea. Clinical course is variable with some patients requiring immunosuppressive therapy. Autoimmune disorders are common and an immune dysregulation mechanism is probably responsible for most cases. The reason for the sparing of the duodenum in some cases is unclear.

\section{LIPONEUROCYTOMA WITH UNUSUAL FEATURES}

Emilia Gottberg, Fouzia Ziad

Waikato Hospital, Department of Histology and Cytology, New Zealand

Cerebellar liponeurocytoma is a fairly rare adult CNS neoplasm though to have a good prognosis. It occurs most often in the cerebellum but supratentorial cases have been reported. The WHO classification and grading of this tumour is currently under neuronal and mixed neuronal-glial tumours and the grade is II, as increasing numbers of reports of unusual features have been found and follow up of earlier cases have shown a recurrence rate of up to $60 \%$. This neoplasm has previously been known under several different names: lipomatous medulloblastoma, lipidized medulloblastoma, medullocytoma, lipomatous glioneurocytoma and lipidized mature neuroectodermal tumour of the cerebellum. In $2000 \mathrm{WHO}$ adopted the term cerebellar liponeurocytoma.

The lesion is composed of neurocytic cells with a varying degree of lipidization, this feature of lipid laden cells resembling adipocytes is the hallmark of liponeurocytomas.

\section{GLIOSARCOMA WITH A PRIMITIVE NEUROECTODERMAL TUMOUR COMPONENT}

C. Green, B. Dessauvagie, P. Robbins

Pathwest, Sir Charles Gairdner Hospital, Australia

Background: Gliosarcomas are rare and highly aggressive biphasic malignancies containing a mix of gliomatous and mesenchymal components. Gliosarcoma represents $2-8 \%$ of glioblastomas, with no influence on clinical course or prognosis in comparison to glioblastoma of no special type.

CNS primitive neuroectodermal tumour (PNET) is defined as a predominantly neuronal but multi-potential non-cerebellar embryonal neoplasm with medulloblastoma-like histology. Adult supratentorial PNETs are well recognised CNS tumours with combined features of malignant glioma and primitive neuroectodermal tumour (MG-PNET). These are rare and poorly characterised, providing difficulties in diagnosis and therapeutic approach.

The PNET component of MG-PNET commonly consists of sharply demarcated nodules which are hypercellular and show evidence of neuronal differentiation. The PNET component is believed to arise in pre-existing gliomas, most often glioblastoma of 'secondary' type, rather than representing either a PNET with extensive glial differentiation or a 'collision' tumour. The clinical implications of this diagnosis include increased rates of CSF seeding and possible response to platinum based chemotherapies, a therapeutic approach not typically used in the treatment of adult glioblastoma or gliosarcoma.

Objectives: To discuss a rare entity with potential for misdiagnosis and implications for treatment.

Case description: 55-year-old man with 4 weeks of headaches and right temporal lobe lesion on CT head. Histologically the glioma demonstrated a complex mixture of patterns, with conventional GBM, areas of gliosarcoma, and distinct nests of PNET. A diagnosis of gliosarcoma with PNET component, the patient received platinum based chemotherapy and cranio-spinal radiotherapy.

Conclusion: CNS tumours with combined elements of a malignant glioma and PNET are both rare and poorly characterised, 